\title{
A 3D-Collaborative Wireless Network: Towards Resilient Communication for Rescuing Flood Victims
}

\author{
Md Arafatur Rahman \\ Faculty of Computer System \\ and Software Engineering \\ University Malaysia Pahang \\ and IBM Center of Excellence \\ Pahang, Malaysia_Email: monirul.iiuc@gmail.com \\ E-mail: arafatur@ump.edu.my \\ Md Munirul Hasan \\ Faculty of Computer System \\ and Software Engineering \\ University Malaysia Pahang \\ Pahang, Malaysia.
}

\author{
A. Taufiq Asyhari \\ Centre for Electronic Warfare \\ Information and Cyber, \\ Cranfield University,
}

\author{
Md Zakirul Alam Bhuiyan \\ Department of Computer \\ and Information Sciences \\ Fordham University, JMH
}

Defence Academy Shrivenham, 328A, Bronx NY, 10458 USA.

SN6 8LA, UK

Email: taufiq-a@ieee.org

\begin{abstract}
Every year, floods result in huge damage and devastation both to lives and properties all over the world. Much of this devastation and its prolonged effects result from a lack of collaboration among the rescue agents as a consequence of the lack of reliable and resilient communication platform in the disrupted and damaged environments. In order to counteract this issue, this paper aims to propose a three-dimensional (3D)collaborative wireless network utilizing air, water and ground based communication infrastructures to support rescue missions in flood-affected areas. Through simulated Search and Rescue(SAR) activities, the effectiveness of the proposed network model is validated and its superiority over the traditional SAR is demonstrated, particularly in the harsh flood environments. The model of the 3D-Collaborative wireless network is expected to significantly assist the rescuing teams in accomplishing their task more effectively in the corresponding disaster areas.
\end{abstract}

Keywords-Collaborative wireless network, flood rescuing system, Search and Rescue (SAR), resilient network.

\section{INTRODUCTION}

According to a report published by the Centre for Research on the Epidemiology of Disasters, approximately 388 natural disasters occurred annually from 2003 to 2012, which had caused the death of 106,654 people on annual average [1]. The same report also highlighted a much higher figure of disaster victims, reaching an annual mean of 216 million people worldwide. Among all the natural disasters, flooding accounts for more than its fair share of fatalities, showing statistically significant values. One of the challenges when dealing with floods is in the post-flood rescue and relief activities, where coordination of activities via air, ground and water-based vehicles requires a costly investment in modern networks and communications infrastructure, which is often not practical given the hostilities of the anticipated communication environments and remoteness of the coverage areas. As a result, the lack of communications and data relay hampers effective rescue activities and extends the effects of the flood, magnifying the underlying death toll and significantly increasing the number of those displaced.

An effective Search and Rescue (SAR) system can assist the rescuers in finding the victims efficiently. The design

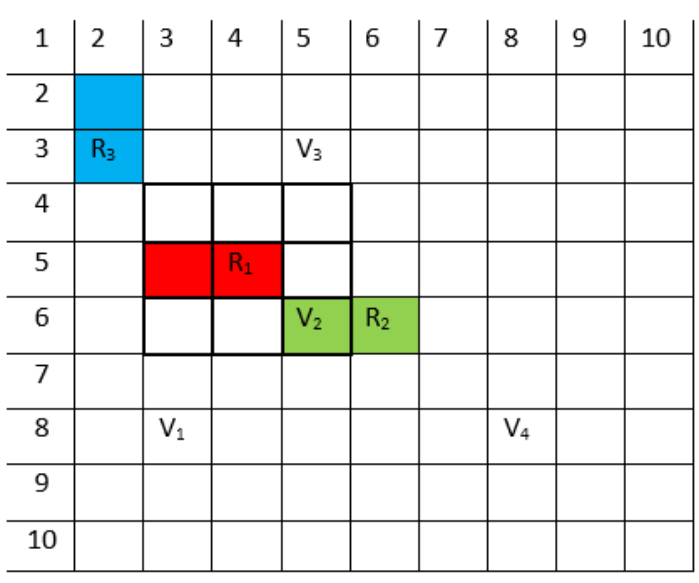

$\mathrm{R}=$ Rescuer, $\mathrm{V}=$ Victim and Shaded box $=$ Visited place

Fig. 1: The impact of collaboration on flood rescuing system.

of a SAR system is more challenging than others because of the harshness of the environment. In order to design an efficient SAR system for such a scenario, establishing seamless communication among the rescuers is one of the essential features. Due to a typical lack of this feature, the rescuer might visit an area that is already being searched by another rescuer, which reduces the efficiency of the SAR system. The prime goal of the SAR system is to rescue the victims as quickly as possible in order to save their lives. The system should be designed to depend on not only the single object observation but also multi-object observations. For clarity, we illustrate this issue in Fig. 1 where there are 3 rescuers and 4 victims located in a disaster area of size $[10 \times 10]$ grids. If the first rescuer, denoted as $R_{1}$, visits to his next grid $(6,5)$ based on his own observation, it will not be an efficient move, since $R_{2}$ already rescued the victims $V_{2}$. Therefore, it is important to have collaboration among the rescuers in order to take efficient move for the next grid. Moreover, the rescuers are not able to visit all the grids because of the harshness of the environment, i.e., some areas are covered by water or jungle and as a result, 
the rescuers cannot directly exchange information reliably. In order to access all the grids, three dimensional collaborative efforts are required, i.e., incorporating air-, water- and groundlevel collaboration. Both one and two dimensional models have been proposed for a variety of applications [9]-[16], however, $3 \mathrm{D}$ collaboration for such a disaster-rescue scenario has not been well addressed in the existing literature.

Enabling resilient communication to facilitate collaboration among the rescue agents is therefore crucial for developing an effective rescuing system. In support for this aim, this paper proposes a three-dimensional-collaborative wireless network $(3 D-C W N)$ utilizing air-, water- and ground-based communication infrastructures to support rescue missions in the flood affected areas. The effectiveness of this collaborative network is validated through simulated SAR activities. The proposed SAR-exploiting collaborative network architecture is then shown to outperform the traditional SAR in terms of the number of rescued victims, particularly in harsh flood environments where heavy rains and damaged water-covered areas are prevalent.

The rest of the paper is organized as follows. In Section II, we review existing works. Section III describes in detail the proposed 3D-CWN, comprising its components, architecture and supporting technologies, in alignment of its envisaged application in a flood rescuing system (FRS). In Section IV, we validate the proposed 3D-CWN through simulated SAR activities. Section V concludes the paper by summarizing the main findings.

\section{RELATED WORKS}

Extensive works on designing infrastructure for wireless mesh, and ad-hoc networks have been presented in [2]-[11]. For instance, an infrastructure for wireless mesh networks and a wireless communication network for advanced metering have been proposed in [2], [3] and [4], respectively. Similarly, ad-hoc networks infrastructure have been proposed for performing search and rescue operations in [5] and [6]. Another infrastructure for remote environmental monitoring systems has been proposed in [9]. One major drawback of these wireless infrastructures is the compatibility in the hostile communication environments (e.g., flood affected areas) due to their limited communication range.

The issue of designing infrastructure using sensors for enabling communication among ground vehicles has been considered in a number of works. In references [12]-[14], a reliable intra-vehicular communication infrastructure using ZigBee was proposed. However, due to the limited vicinity and bandwidth of sensor nodes, this proposed infrastructure is unable to assure any degree of quality of service (QoS) necessary for post-flood SAR scenario.

All the aforementioned infrastructures are not collaborative networks. There exist a number of works that consider communication infrastructures for Unmanned Air Robots (UAR) [20], [21]. A high-level description of the issues, opportunities and future research challenges of UARs are presented in [20]. These infrastructures are not fully suitable for comprehensive post-flood rescue activities since they only address wireless networks for aerial vehicles. The wireless infrastructure that aligns well with human rescue and monitor missions in the flood affected area has to consider three degrees of challenges corresponding to the air-, ground- and water-based communication networks. In [22], a prototype for a flood monitoring system (FRS) has been proposed based on the synthetic aperture radar data.

In reference [15], a collaborative sensor network has been proposed for crop monitoring. Due to the limited-range and limited-bandwidth of the sensor nodes, it is not directly compatible in the flood rescue application. In references [16] and [17], two other network infrastructures have also been proposed for urban environment and surveillance network, respectively. There are also works that consider air-ground collaboration for alpine communications as discussed in [18], [19]. Further collaborative wireless networks could be found in the literature encompassing a variety of applications such as file delay minimization for content uploading to media cloud [23], an eavesdropping attack [27], a near-optimal packet allocation algorithm for content uploading to media cloud [28], a store-and-delivery based media access control for precision agriculture [29] and a dynamic self-calibration [30]. However, since the communication issues, challenges, and network components addressed in those works are application-specific, their proposed collaborative infrastructures are neither directly portable to support post-flood SAR activities. Moreover, in this work we specifically propose to utilize three-dimensional collaboration of network components spread over air, ground and water surface to ensure smooth, rapid and effective rescue actions during the flood events.

\section{3D-COLlaborative WiRELESS NETWORK IN FLOOD RESCUING SYSTEM}

3D-Collaborative Wireless Network (3D-CWN) is a network that lies in the three space domains (air, land, water) whereby each network element communicates with one another in order to accomplish a set of agreed tasks. In this work we aim to utilize the 3D-CWN for a specific application in the flood rescuing system (FRS), which aims to retrieve people in distress, provide for initial medical needs and transport them to a safe place. One of the largest challenges for such a system is to establish a resilient communication platform that enables rapid and reliable exchange of critical information. We envisage to achieve this resiliency using 3D-CWN by exploiting the features of: i) collaborative network elements, ii) diversification of supporting communication technologies exploiting Cognitive Radio (CR) and Software Defined Network (SDN) (see Section III-A7 and Table I).

\section{A. Network Components}

The collaborative wireless network for the FRS comprises multiple components, namely, Land Rover (LR), Water Rover (WR), Long Range Manned Air Vehicle (LRMAV), Short Range Unmanned Air Vehicle (SRUAV), Human Rescuer (HR), Regional Control Center (RCC) and Control Center (CC). A collaborative efforts of these components would assist in responding rapidly and accurately in the flood victims' rescuing missions. Roles and tasks of these components are discussed in the following. 


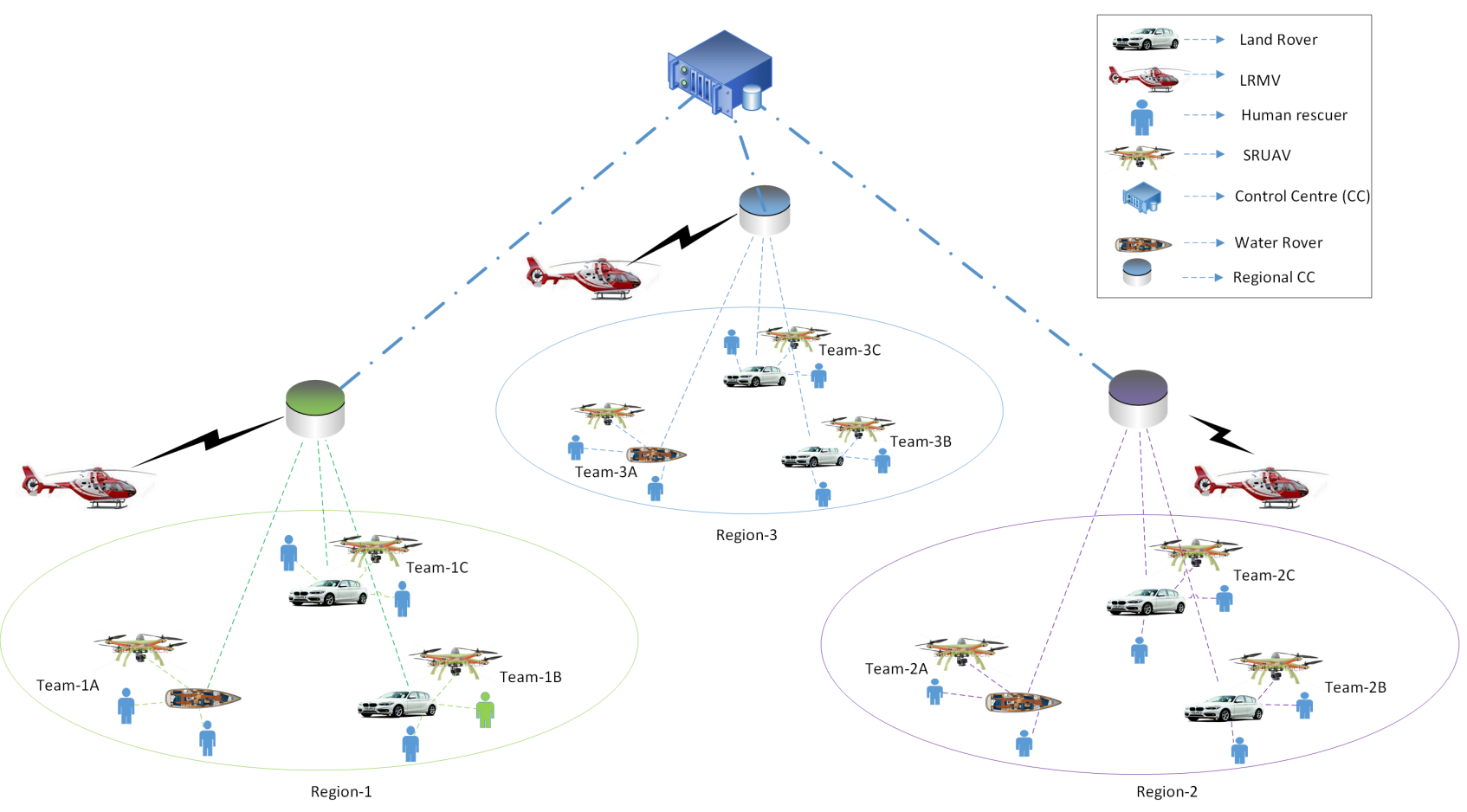

Fig. 2: Overview of 3D-CWN for FMRS.

1) Human Rescuer: A human rescuer is an expert of a specific rescuing mission and has specialist cognitive abilities. They are involved in the direct rescue mission. The rescuers are able to go to the door of victims and rescue them if necessary. They can carry Portable Communication Equipments (PCEs) to communicate with their team through an access point, which is installed in the land rover or water rover. The success of the rescue mission depends on the rescuer efficiency and responsiveness.

2) SRUAV: The objectives of this aerial vehicle are to collect information from the flood affected area and send it to the human rescuers. It is decorated with high exploration cameras and transceivers, which are used to support for collecting and forwarding information in the disaster area. It functions as extended flying eyes of the rescuer which assist in monitoring and searching over the surrounding environment. The ability of SRUAV is to fly very closely to the ground for a closer micro detection of victims in the flood area as well as highly movable and agile in its corresponding territory.

3) Water Rover: The water rover specifically works together with the rescuers and SRUAV to rescue victims in the water-flooded area. It is equipped with a communication equipment that enables exchange of information with other teams and regional control command. The rover team collect information through the use of SRUAV and take actions based on the acquired messages. The water rover is able to work in both shallow water and fast flowing water conditions.

4) Land Rover: The primary objective of the land rover is to rescue in a shallow flood water condition and muddy areas. It is wirelessly connected to the rescuers, so that it is able to follow their movement. Like the water rover, it carries SRUAV to perform a searching task and exchange of information with the regional control center when it needs any equipment supply or assistance.

5) LRMAV: The objective of LRMAV is to search victims by utilizing long-range and high-resolution cameras and to carry compulsory materials such as pure water, foods and first aid box for the victims. Hence, it has a versatile capability that allows to round patrol for a large area with a remarkable payload and ability to fly in difficult weather and geographical conditions. It is therefore able to visit over the isolated remote villages that are not accessible by other vehicles. The operation of LRMAV is directly controlled by the regional control center, which communicates information to all the relevant teams in the case of critical emergency support such as sending victims to a hospital.

6) Regional Control Center (RCC): The RCC acts as a central point of all teams in a given region, which is a subset of the overall flood affected area. The assignment of the RCC is to acquire rapid update of rescuing activities from all the teams in the corresponding region and supply any required assistance. Another task of the RCC is to control the LRMAV and also maintain contact with the control center in order to exchange information about the flood situation and collect necessary equipments, medicals and tools from the control center.

7) Control Center (CC): In our proposed 3D-CWN for the FRS, there exists only one CC, which acts as the head of the rescue mission and has ultimate authority over all the units. The CC controller has significant experience with regard to the flood situation. Its main role is to receive information from various RCCs and, according to the information, will provide direction, advice, relief supplies and equipments to the rescue teams. The central ministries, media and various organizations retrieve updates from the CC. 


\begin{tabular}{|c|c|c|c|c|c|c|c|}
\hline Technologies & Frequency & Maximum Data Rate & Coverage & Mobility & Low Latency & Encryption & Rain Affect \\
\hline WiFi & $\begin{array}{l}2.4-2.4835 \mathrm{GHz} \\
80 \mathrm{MHz}\end{array}$ & $\begin{array}{l}150 \mathrm{Mbps} \\
5.15-5.35 \mathrm{GHz} \\
6.77 \mathrm{Mbps}\end{array}$ & $\begin{array}{l}250 \mathrm{~m} \\
\text { around } 250 \mathrm{~m}\end{array}$ & Yes & Yes & WEP, WPA, WPA2 & $\begin{array}{l}\text { Less } \\
\text { Less }\end{array}$ \\
\hline ZigBee & $2.4 \mathrm{GHz}$ & $\begin{array}{l}250 \mathrm{kbps} \\
915 \mathrm{MHz} \text { in US } \\
2.4 \mathrm{GHz} \text { Worldwide }\end{array}$ & $\begin{array}{l}10-150 \mathrm{~m} \\
40 \mathrm{kbps} \\
250 \mathrm{kbps}\end{array}$ & Yes & No & 128-bit AES & Less \\
\hline XBee & $2.4 \mathrm{GHz}$ & $250 \mathrm{kbps}$ & $1.6 \mathrm{~km}$ & Yes & No & 128-bit AES & Less \\
\hline WiMAX & $\begin{array}{l}10-66 \mathrm{GHz} \\
2-11 \mathrm{GHz} \\
2-6 \mathrm{GHz}\end{array}$ & $\begin{array}{l}32-134 \mathrm{Mbps} \\
\text { up to } 75 \mathrm{Mbps} \\
\text { up to } 15 \mathrm{Mbps}\end{array}$ & Up to 30 miles & Yes & Yes & PKMV2, EAP, EAS & $\begin{array}{l}\text { High } \\
\text { High } \\
\text { High }\end{array}$ \\
\hline LTE & $1-3 \mathrm{GHz}$ & $\begin{array}{l}\text { Downlink } 300 \mathrm{Mbps} \\
\text { Uplink } 75 \mathrm{Mbps}\end{array}$ & $5 \mathrm{~km}$ & Yes & Yes & SNOW 3G Stream Cipher & Less \\
\hline
\end{tabular}

TABLE I: Comparison among the existing wireless technologies.

\section{B. Architecture}

Fig. 2 depicts the network architecture of the FRS. This architecture features: i) information acquisition; ii) information forwarding and storing; and iii) victims rescue and first aid provider. The objective of the first feature is to acquire victims' information from the flood affected area. Two types of communications are designed for information acquisition i.e., Intra-Team and Inter-Team communications. It can be observed from Fig. 2 that the searching activities are conducted by the rescue teams and LRMAV. Each team consists of the HRs, SRUAV and LR/WR. Information is shared within a team through the LR/WR and local wireless connection. Each team can then exchange information with one another through the LR/WR and RCC. Both Intra-Team and Inter-Team communications can help to collect information efficiently in the FRS. The objective of the second feature is to forward the collected information to the upper levels of RCC and CC in order to store the information. This task can be accomplished by interconnecting the LR/WR, RCC and $\mathrm{CC}$. The stored information can then be further utilized by the teams and LRMAVs so that an efficient victims searching mechanism can be designed. The objective of the third feature is to rescue the victims by exploiting the first two features and supply them with the essential first aid kit. The HRs and LR/WR provide support to achieve this goal. The collaborative efforts of all these network components can significantly improve the performance of SAR activities, to be discussed in Section IV.

\section{Technologies in CWN for FRS}

In this section we will assess and select compatible wireless technologies for communication in the flood environments. Selection consideration includes transmission coverage, data rate, mobility, latency, security and signal attenuation due to expected heavy rainfall. Table I summarizes the comparison of existing wireless technologies by addressing the important characteristics. Since the flood affected area is in terms of $\mathrm{km}$ scale, high transmission coverage is therefore required. In this context, 802.16/WiMAX and LTE are suitable candidates whereas the others are less preferable. During the rescuing time, we need to take real time pictures and videos and also send them to the rescuers. Consequently, high data rate and low latency features are preferable, which can be facilitated by $\mathrm{WiFi}$, WiMAX and LTE. Mobility support is another important feature that can provided by all the aforementioned existing technologies. Security plays a crucial role for technology selection. The encryption techniques of the different technologies are mentioned in Table I. ZigBee, XBee, WiMAX and LTE utilize more secure encryption techniques compared to WiFi. In the scenario of mobile communications over the flood environment, the integrity of wireless signals is subject to channel fading, which depends not only on the timevarying multipath propagation [24], [25], but also rain intensity [26]. While the former factor can be addressed through, e.g., utilization of forward error correction mechanisms in the existing technologies in Table I, the latter factor is frequencydependent. Raindrops can severely corrupt communication signals for frequency above $5 \mathrm{GHz}$ frequency band. From this perspective, WiMAX seems to be less suitable for the flood environment.

Based on the above discussion and summary in Table I, LTE appears to be a strong candidate for the flood environment compared to other technologies. However, relying solely on LTE might not fully offer communication resilience as it may fail due to the hostile communication environment. Diversification of supporting communication technologies exploiting the concept of CR and SDN is a sensible approach to mitigate a single point of failure. In order to make the network more resilient, each device (e.g., PCE, SRUAV, land and water rovers) will have cognitive radio enabled so that it will be able to communicate with one another using flexible multiple spectrum bands that exploit the SDN concept. In emergency cases (i.e., when LTE fails), the devices can communicate through XBee and WiFi and share the rescue information through multi-hop communications.

\section{VAlidation of the Proposal}

In this section, we validate the effectiveness of the proposed collaborative network through simulated SAR activities, which incorporate search for and provision of aid to the people in suffering or imminent danger. For the purpose of benchmarking and comparison, we categorize SAR into three types: i) one dimensional SAR (1D-SAR), i.e., if the rescuers are able to search only the Land Covered Area (LCA), referred to as the traditional SAR; ii) two dimensional SAR (2D-SAR), i.e., if the rescuers are able to search both the LCA and Water Covered Area (WCA); iii) three dimensional SAR (3DSAR), i.e., if the rescuers are able to search not only the LCA and WCA but also the Critical Area (CA), which is an area where only drone is capable to reach for performing the search activities. The proposed collaborative network is designed to perform the 3D-SAR. Fig. 3 depicts the simulated flood affected region where the area is $10^{2} \mathrm{~km}$. The whole area is covered by all the LCA, WCA and CA. We carry 


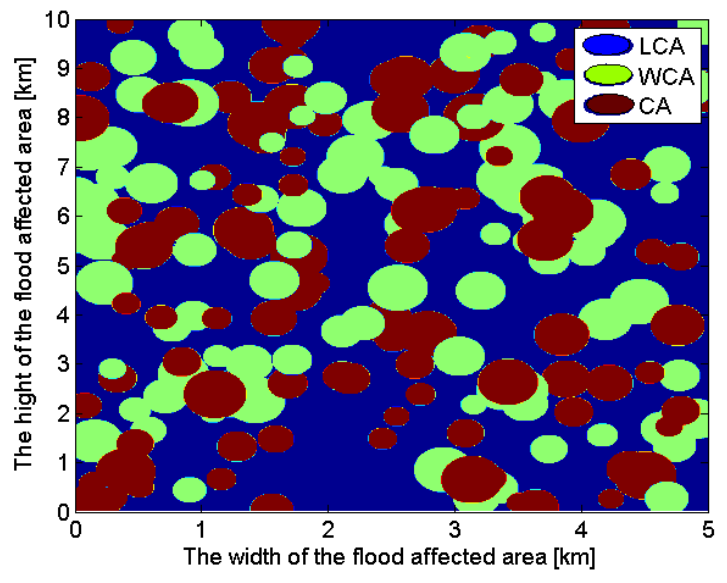

Fig. 3: The affected area covered by LCA, WCA and CA during the flood.

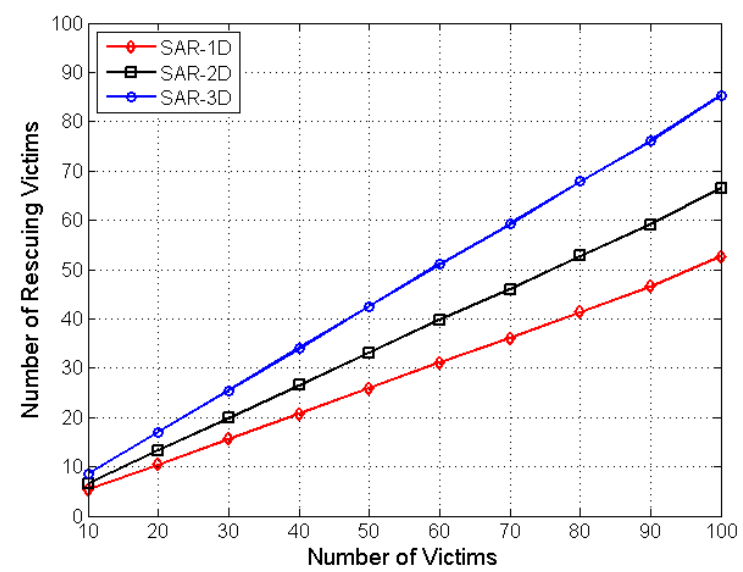

Fig. 4: The efficiency of the rescuing activity by 1-D, 2-D and 3-D SAR.

out experiments in order to validate the effectiveness of our proposal and the results are discussed in the following.

In Fig. 4, we measure the performance of 1D-SAR, 2DSAR and 3D-SAR with respect to the number of rescued victims from the number of victims in the scenario. The simulation set is as follows: the area is $10^{2} \mathrm{~km}$; the number of victims set $\vartheta=\{10,20,30,40,50,60,70,80,90,100\}$ is randomly placed in the flood affected area; the number of WCA and CA are 100, which are randomly placed inside the network area; the depths $\delta$ of the WCA and CA are chosen randomly from $200 \mathrm{~m}$ to $500 \mathrm{~m}$. It can be observed that, by using the 1D-SAR, we are not able to rescue the victims who are in the WCA and CA regions, since they are not searchable by the $1 \mathrm{D}-\mathrm{SAR}$ enabled rescuers. By using the 2D-SAR, we are able to rescue more than that using the $1 \mathrm{D}-\mathrm{SAR}$ but not the victims who are in the CA region. By exploiting the 3D$\mathrm{SAR}$, we are able to rescue more victims compared to $2 \mathrm{D}$ and $1 \mathrm{D}-\mathrm{SAR}$. In this case, the 3D-SAR seems to outperform the others because all the disaster area can be accessed either by the land rover, water rover, LRMAV or SRUAV.

If the number of WCA and CA increase, the flood affected area will be more severe. In this case, the performances of the 1D-SAR and 2D-SAR degrade more compared to the 3D-SAR,

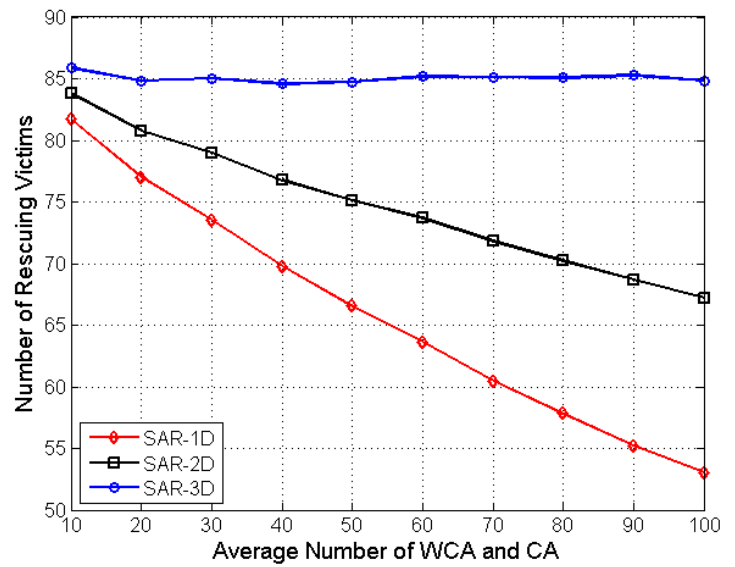

Fig. 5: The effectiveness of the rescue mission varies with the number of WCA and CA.

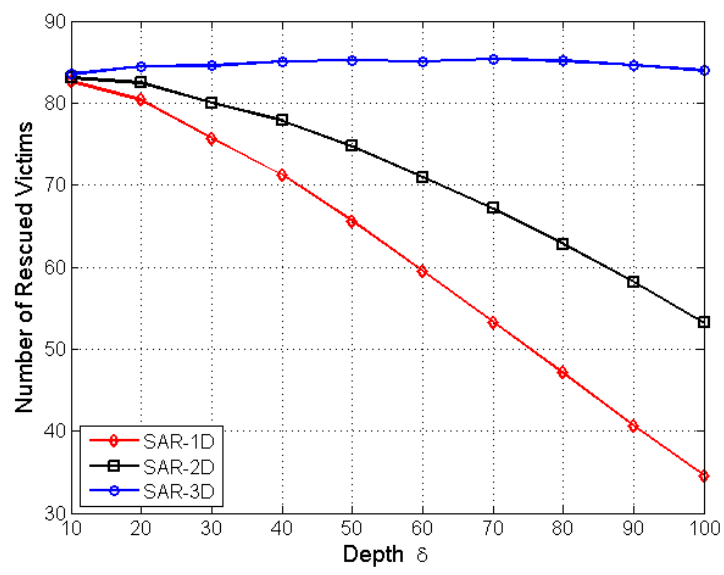

Fig. 6: The effectiveness of the rescue mission varies with the depth of WCA and CA.

as depicted in Fig. 5. In this experiment, the simulation setup is mostly similar to that in Fig. 5 with the following minor differences. The number of victims in the scenario is 100 and the sets of average number of WCA and CA are both given by $\{10,20,30,40,50,60,70,80,90,100\}$. We notice that, by increasing the number of WCA and CA for a given area, the LCA is effectively reduced, and as a result, the number of victims are less in the LCA. In this case, the $1 \mathrm{D}-\mathrm{SAR}$ is only able to rescue less victims compared to the 2D- and 3D-SAR. The flood affected area is also more severe if the average depths $\delta$ of WCA and CA increase. In this case, by following a similar line of reasoning to that in Fig. 5, the performances of the 1D-SAR and 2D-SAR deteriorate more than that of the 3D-SAR, as shown in Fig. 6.

These results demonstrate that the proposed network model offers promising characteristics and potential to improve the effectiveness of the SAR activities in the flood affected area.

\section{CONCLUSION}

We have proposed a 3D-collaborative network model for rescuing the victims in flood-affected areas. The proposed 
network model can assist in acquiring victims' information, forwarding and storing the collected information, and developing follow-up rescue actions. The effectiveness of the proposed network has been validated through simulated SAR activities. More specifically, our experimental results have demonstrated that the 3D-collaborative efforts of the network components offer more resilient communication platform in harsh flood environments, which in turn can significantly improve the number of rescued victims in the simulated SAR activities. The proposed model can serve as a basis for the development of an efficient searching-activity system, which will be considered in the future direction of this work.

\section{ACKNOWLEDGMENTS}

This work is partially supported by the University Malaysia Pahang under the projects "RDU1603129" and "RDU160360".

\section{REFERENCES}

[1] D. Guha-Sapir, P. Hoyois and R. Below, "Annual disaster statistical review 2013: The numbers and trends," Centre for Research on the Epidemiology of Disasters (CRED), Brussels, 2014.

[2] Z. Weiyi and X. Jiang, "ReLoAD: resilient location area design for internet-based infrastructure wireless mesh networks," in Proc. IEEE Global Telecommunications Conference (GLOBECOM 2011), Kathmandu, 2011.

[3] M. A. Rahman, M. S. Azad, F. Anwar, and A. H. Abdalla, "A simulation based performance analysis of reactive routing protocols in wireless mesh networks," in Proc. 2009 International Conference on Future Networks, Bangkok, 2009.

[4] M. A. Rahman, M. S. Azad and F. Anwar, "Integrating multiple metrics to improve the performance of a routing protocol over wireless mesh networks," in Proc. International Conference on Signal Processing Systems, Singapore, 2009.

[5] R. G. L. Narayanan and O. C. Ibe, "A joint network for disaster recovery and search and rescue operations," Computer Networks vol. 56, pp. 33473373, 2012.

[6] F. Anwar, M. Azad, M. Rahman, and M. M. Uddin, "Performance analysis of ad hoc routing protocols in mobile WiMAX environment,' IAENG International Journal of Computer Science vol. 35, no. 4, pp. 353-360, 2008.

[7] C. Ameixieira, A. Cardote, F. Neves, R. Meireles, S. Sargento, et al., "Harbornet: A real-world testbed for vehicular networks," IEEE Communications Magazine, vol. 52, no. 9, pp. 108-114, Sep. 2014.

[8] J. Lloret, A. Canovas, S. Sendra and L. Parra, "A smart communication architecture for ambient assisted living," IEEE Communications Magazine, vol. 53, no. 1, pp. 26-33, 2015.

[9] Y. F. Yang, V. Gondi, J.O. Hallstrom, W. Kuang-Ching, G. Eidson and C.J. Post, "Wireless infrastructure for remote environmental monitoring: Deployment and evaluation," in Proc. IEEE International Conference on Mobile and Wireless Networking (MoWNeT), Montreal, 2013.

[10] X. Li, B. j. Hu, H. Chen, B. Li, H. Teng and M. Cui, "Multi-hop delay reduction for safety-related message broadcasting in vehicle-to-vehicle communications," IET Communications, vol. 9, no. 3, pp. 404-411, 2015.

[11] A. Adebanjo, A. M. Lanre, E. J. Bamidele, A. C. Emmanuel, O. N. Camilia, A. B. Kamalrulnizam, "An evaluation of improved cluster-based routing protocol in ad-hoc wireless network," International Journal of Software Engineering and Computer Systems, vol. 3, no. 3, pp. 146157, 2017.

[12] M. A. Rahman, "Reliability analysis of ZigBee based intra-vehicle wireless sensor networks," in Proc. International Workshop on Communication Technologies for Vehicles, Offenburg, 2014.

[13] M. A. Rahman, "Design of wireless sensor network for intra-vehicular communications," in Proc. International Conference on Wired/Wireless Internet Communications, Paris, 2014.

[14] A. White, "A collaboration network for unmanned aerial vehicle operation," DTIC Document, 2005.
[15] G. Tuna, T. V. Mumcu, K. Gulez, V. C. Gungor, and H. Erturk, "Unmanned aerial vehicle-aided wireless sensor network deployment system for post-disaster monitoring," in Proc. International Conference on Intelligent Computing, Huangshan, 2012.

[16] J. Valente, D. Sanz, A. Barrientos, J. D. Cerro, Ribeiro, and C. Rossi, "An air ground wireless sensor network for crop monitoring," Sensors, vol. 11, no.6, pp. 6088-6108, 2011.

[17] L. Chaimowicz, A. Cowley, D. Gomez-Ibanez, B. Grocholsky, M. A. Hsieh, H. Hsu, et al., "Deploying air-ground multi-robot teams in urban environments," Multi-Robot Systems. From Swarms to Intelligent Automata, vol. 3, pp. 223-234, 2005.

[18] M. Dille, B. Grocholsky, S. T. Nuske, M. Moseley and S. Singh, "Air-ground collaborative surveillance with human-portable hardware," 2011. [Online]. Available: http://repository.cmu.edu/cgi/viewcontent.cgi?article=1937\&context= robotics/.

[19] M. A. Rahman, "Enabling drone communications with WiMAX technology," in Proc. The 5th International Conference on Information, Intelligence, Systems and Applications, IISA 2014, Chania, Greece, 2014.

[20] M. A. Rahman, "SHERPA: An air-ground wireless network for communicating human and robots to improve the rescuing activities in alpine environments," in Proc. International Conference on Ad-Hoc Networks and Wireless, Benidorm, Spain, 2014.

[21] Y. Saleem, M. H. Rehmani and S. Zeadally, "Integration of cognitive radio technology with unmanned aerial vehicles: Issues, opportunities, and future research challenges," Elsevier Journal of Network and Computer Applications, vol. 50, pp. 15-31, 2015.

[22] G. Boni, L. Ferraris, L. Pulvirenti, G. Squicciarino, N. Pierdicca, et al. "A prototype system for flood monitoring based on flood forecast combined with COSMO-skymed and sentinel-1 Data," IEEE Journal of Selected Topics in Applied Earth Observations and Remote Sensing, vol. 9, no.6, pp. 2794-2805, 2016.

[23] G. Zhang, Y. Wen, J. Zhu and Q. Chen, "On file delay minimization for content uploading to media cloud via collaborative wireless network," in Proc. IEEE International Conference on Wireless Communications and Signal Processing (WCSP), Nanjing, 2011.

[24] A. T. Asyhari, T. Koch, and A. Guillén i Fàbregas, "Nearest neighbour decoding with pilot-assisted channel estimation for fading multipleaccess channels," in Proc. The 49th Annual Allerton Conference on Communication, Control, and Computing, Monticello, IL, 2011.

[25] A. T. Asyhari and A. Guillén i Fàbregas, "MIMO block-fading channels with mismatched CSI," IEEE Transactions on Information Theory, vol. 60, no. 11, Pages 7166-7185, 2014.

[26] A. G. Kanatas and A. D. Panagopoulos, Radio Wave Propagation and Channel Modeling for Earth-Space Systems. Boca Raton, FL: CRC Press, 2016.

[27] Y. Zou, X. Wang and W. Shen, "Eavesdropping attack in collaborative wireless networks: Security protocols and intercept behavior," in Proc. The 17th IEEE International Conference on Computer Supported Cooperative Work in Design (CSCWD), Whistler, BC, 2013.

[28] G. Zhang, Y. Wen and Y. Ong, "Near-optimal packet allocation algorithm for content uploading to media cloud via collaborative wireless network," in Proc. IEEE International Conference on Computing, Networking and Communications (ICNC), San Diego, CA, 2013.

[29] S. Liew, S. Azad, H. G. Goh, B. Y. Ooi and M. A. Rahman, "A storeand-delivery based MAC protocol for air-ground collaborative wireless networks for precision agriculture," in Proc. The 21st IEEE International Conference on Parallel and Distributed Systems (ICPADS), Melbourne, 2015.

[30] Y. Zhang and H. Dai, "Dynamic self-calibration in collaborative wireless networks using belief propagation with gaussian particle filtering," in Proc. The 41st IEEE Annual Conference on Information Sciences and Systems (CISS'07), Baltimore, MD, 2007.

[31] A.S. Cacciapuoti, M. Caleffi, L. Paura and M.A. Rahman, "Channel availability for mobile cognitive radio networks," Elsevier Journal of Network and Computer Applications, vol. 47, pp. 131-136, 2015. 